\title{
Visiones de estudiantes de secundaria sobre Naturaleza de la Ciencia en ambientes de discusión, cuando se incorporan biografías a la clase de ciencias
}

\author{
Luigi Cuellar Fernández \\ Departamento de Didáctica, Facultad de Educación, Universidad Católica de la Santísima Concepción. \\ Concepción.Cbile.lcuellan@ucsc.cl
}

Ainoa Marzábal Blancafort

Departamento de Didáctica. Facultad de Educación. Pontificia Universidad Católica de Cbile. Santiago de Chile.Chile.amarzabal@uc.cl

[Recibido: 5 noviembre 2019. Revisado: 16 marzo 2020. Aceptado: 12 mayo 2020]

\begin{abstract}
Resumen: En este trabajo de investigación caracterizamos las visiones sobre la Naturaleza de la Ciencia de estudiantes en cuyas clases se incorporan aspectos históricos desde una perspectiva biográfica, asumiendo que proporcionan una experiencia escolar con potencial para transformar las visiones ingenuas que persisten actualmente en el sistema escolar. El análisis cualitativo de los datos de cuatro casos de estudio, obtenidos mediante cuestionario y grupo focal, nos permitieron caracterizar las visiones sobre Naturaleza de la Ciencia predominantes de los estudiantes. Los resultados apuntan a que los estudiantes participantes identifican claramente los aspectos epistémicos, reconociendo el conocimiento científico como un conjunto de representaciones de los científicos sobre el mundo natural de carácter subjetivo y tentativo. Sin embargo, no logran establecer con claridad aspectos no epistémicos como el rol de la negociación social ni el impacto cultural en el desarrollo de la ciencia, al situar la actividad científica en una dimensión personal más que social. Esto evidencia la necesidad de avanzar hacia la visibilización del carácter controversial, culturalmente diverso y socialmente situado de la ciencia en las biografías científicas escolares, y de la actividad científica como una práctica académica, para que esta estrategia didáctica tenga mayor potencial para contribuir a una comprensión holística de la Naturaleza de la Ciencia.
\end{abstract}

Palabras clave: Educación escolar, Biografías científicas, Naturaleza de la Ciencia, Alumnado.

High school students' views of Nature of Science in discussion settings, when biographies are incorporated into science class

\begin{abstract}
In this research work we characterize the Nature of Science views of students in whose science classes historical aspects are incorporated from a biographical perspective, assuming that they provide a school experience with the potential to transform the positivist visions that persist in schools. The qualitative analysis of the data from four case studies, obtained through a questionnaire and focus group, allowed us to characterize the predominant students' Nature of Science views. The results suggest that participating students clearly identify the epistemic aspects, considering scientific knowledge as a subjective and tentative set of representations of scientists about the natural world. However, they are not able to clearly establish non epistemic aspects, as the role of social negotiation or the cultural impact on the development of science, because they conceive scientific activity in a personal rather than a social dimension. This shows the need to move towards the visibility of the controversial, culturally diverse and socially located features of science in school scientific biographies, and of scientific activity as an academic practice, so that this teaching strategy has greater potential to contribute to the students' holistic understanding of the Nature of Science.
\end{abstract}

Keywords: School education, Scientific Biographies, Students, Nature of Science, Students

Para citar este artículo: Cuellar, L. y Marzábal, A. (2020) Visiones de estudiantes de secundaria sobre Naturaleza de la Ciencia en ambientes de discusión, cuando se incorporan biografías a la clase de ciencias. Revista Eureka sobre Enseñanza y Divulgación de las Ciencias 17(3), 3102. doi: 10.25267/Rev_Eureka_ensen_divulg_cienc.2020v17.i3.3102 


\section{Introducción}

El cambio de paradigma epistemológico desde el positivismo lógico al constructivismo en las últimas décadas ha llevado a la reforma de los currículos de ciencia y los enfoques de enseñanza (Tsai 2003). Considerando que una comprensión adecuada sobre la Naturaleza de la Ciencia $(\mathrm{NdC}$ ) es un componente esencial de la alfabetización científica (Duschl 1990; McComas 2008), se han dedicado intensos esfuerzos de investigación a la caracterización de las visiones de los estudiantes (Tsai y Liu 2005). Los resultados de la investigación sugieren que los puntos de vista sobre la $\mathrm{NdC}$ de los estudiantes median en su motivación para aprender ciencias (Dagher y Erduran 2016; Tsai 2003; Cofré et al. 2019) y en la adquisición de conocimiento científico (Songer y Linn 1991).

Actualmente existen diversas propuestas para el desarrollo curricular de la $\mathrm{NdC}$ a nivel escolar (Acevedo-Díaz, García-Carmona y Aragón-Méndez 2017). Sin embargo, los estudios sobre las visiones de profesores y estudiantes siguen reportando comprensiones ingenuas que obstaculizan el aprendizaje de las ciencias experimentales, y la argumentación y la toma de decisiones de los estudiantes sobre cuestiones socio científicas (García-Carmona, Manassero y Vázquez 2012). Los resultados revelan que la mayoría del profesorado de ciencias mantiene una visión tradicional, con una comprensión descontextualizada de la actividad científica, lejos de la vida cotidiana y sin relación con los aspectos históricos y sociales de la ciencia (Cofré y Vergara 2010). Esto dificulta la incorporación explícita de aspectos de NdC en la actividad científica escolar, y contribuye a consolidar la visión positivista de los estudiantes.

En los últimos años hemos abordado la incorporación de la Historia de la Ciencia en la formación de profesores, como un recurso para la comprensión de la $\mathrm{NdC}$ (Cuellar, Quintanilla y Marzabal 2010; Cuellar, Quintanilla y García 2013; Siso y Cuellar 2017; Siso, Sánchez y Cuellar 2019). En nuestros estudios, hemos reportado el fuerte arraigo de las visiones ingenuas en los profesores, y hemos mostrado el potencial de las comunidades de aprendizaje (González-Weil et al. 2014) en las que se incorpora la perspectiva historiográfica, como el contexto en que la transformación de las visiones del profesorado participante es más significativa (Siso y Cuellar 2017). Sin embargo, hasta la fecha, no hemos explorado de qué manera la transformación de las visiones sobre $\mathrm{NdC}$ de los profesores y la incorporación explícita de aspectos históricos en su enseñanza, contribuye al desarrollo de visiones adecuadas sobre la ciencia en los estudiantes.

Han sido necesarios diez años de trabajo continuado para estar en condiciones de explorar las visiones sobre $\mathrm{NdC}$ de estudiantes cuyas profesoras presentan visiones epistemológicas constructivistas coherentes, y que han incorporado progresivamente la historia de la ciencia en sus clases. En este trabajo, nuestro propósito es analizar en profundidad de qué manera la experiencia escolar, a través de los aspectos que se abordan en las clases de ciencias gestionadas por estas profesoras, ha contribuido a configurar las visiones sobre $\mathrm{NdC}$ de sus estudiantes.

\section{Marco teórico}

\section{Aspectos de Naturaleza de la Ciencia relevantes para la Educación Científica}

A pesar del intenso debate entre filósofos, historiadores y sociólogos de la ciencia, todavía existen desacuerdos en la conceptualización de la NdC (Abd-El-Khalick 2012; Acevedo-Díaz y García-Carmona 2016). Sin embargo, ha sido posible llegar a un conjunto de nociones generalizadas y no controversiales sobre la $\mathrm{NdC}$ relevantes para la educación (Dagher y Erduran 2016). Estas nociones consideran aspectos epistémicos de la NdC, centrados en el proceso de construcción de conocimiento científico y sus características, y aspectos no 
epistémicos, centrados en las circunstancias y contextos socioculturales de la ciencia y su desarrollo, avanzando hacia una concepción más holística de la $\mathrm{NdC}$ (Acevedo-Díaz et al. 2017). En cuanto a los aspectos epistémicos, se reconoce que el conocimiento científico es tentativo, cargado teóricamente y creativo, y en cuanto a los aspectos no epistémicos, que se enmarca en procesos de negociación, y que está mediado cultural y socialmente (Tsai 2003). En la tabla 1 sistematizamos las visiones sobre $\mathrm{NdC}$ que emergen desde perspectivas ingenua $\mathrm{y}$ adecuada para cada uno de estos aspectos. Así entonces las visiones sobre $\mathrm{NdC}$ estarían conformadas por las creencias en torno a estos cinco componentes, asumiendo que constituyen creencias que conforman un sistema ecléctico, no necesariamente coherente, en la que pueden identificarse orientaciones dominantes sobre NdC (Vázquez y Manassero 1999).

Tabla 1. Componentes emergentes desde las perspectivas ingenua y adecuada en torno a la Naturaleza de la Ciencia (Elaboración propia a partir de Tsai 2003; Tsai y Liu 2005; Matthews 2017; Dagher y Erduran 2016; Acevedo-Díaz y García-Carmona 2016)

\begin{tabular}{|c|c|c|c|}
\hline & $\begin{array}{l}\text { Visiones sobre la } \\
\text { Naturaleza de la } \\
\text { Ciencia }\end{array}$ & Perspectiva ingenua & Perspectiva adecuada \\
\hline \multirow{3}{*}{$\begin{array}{c}\text { Aspectos } \\
\text { epistémicos }\end{array}$} & $\begin{array}{l}\text { Naturaleza } \\
\text { tentativa del } \\
\text { conocimiento }\end{array}$ & $\begin{array}{l}\text { El conocimiento científico } \\
\text { proporciona explicaciones certeras } \\
\text { sobre el mundo natural, y por } \\
\text { tanto no requieren ser evaluadas } \\
\text { posteriormente. La ciencia } \\
\text { progresa cuando el diseño de } \\
\text { nuevos experimentos, el desarrollo } \\
\text { tecnológico o la detección de } \\
\text { errores en las observaciones o } \\
\text { procedimientos, lleva a desarrollar } \\
\text { ideas, modelos o teorías } \\
\text { complementarios a los anteriores }\end{array}$ & $\begin{array}{l}\text { El conocimiento rientífico } \\
\text { proporciona explicaciones } \\
\text { tentativas sobre el mundo natural, } \\
\text { que están en evaluación } \\
\text { permanente. La ciencia progresa en } \\
\text { la medida en que las ideas, teorías y } \\
\text { modelos son refutadas, ajustadas, } \\
\text { ampliadas o confirmadas por las } \\
\text { investigaciones posteriores, lo que } \\
\text { le da un carácter provisional al } \\
\text { conocimiento científico. }\end{array}$ \\
\hline & $\begin{array}{l}\text { Naturaleza } \\
\text { creativa del } \\
\text { conocimiento }\end{array}$ & $\begin{array}{l}\text { El conocimiento científico es el } \\
\text { resultado del estudio de patrones } \\
\text { en la relación entre variables, } \\
\text { descubiertos por los científicos a } \\
\text { partir del estudio de situaciones } \\
\text { experimentales, que permiten } \\
\text { describir fielmente la realidad. }\end{array}$ & $\begin{array}{l}\text { El conocimiento científico es el } \\
\text { resultado de la interpretación de los } \\
\text { científicos de las situaciones } \\
\text { experimentales, generando } \\
\text { representaciones aproximadas de la } \\
\text { realidad en las que la intuición, la } \\
\text { imaginación, la creatividad e incluso } \\
\text { los sueños y corazonadas juegan un } \\
\text { rol fundamental. }\end{array}$ \\
\hline & $\begin{array}{l}\text { Mediación de la } \\
\text { teoría }\end{array}$ & $\begin{array}{l}\text { Se asume que los científicos } \\
\text { pueden realizar observaciones de la } \\
\text { realidad que no están influenciadas } \\
\text { por otros factores, y por tanto el } \\
\text { conocimiento científico se deriva } \\
\text { de observaciones y procedimientos } \\
\text { objetivos. }\end{array}$ & $\begin{array}{l}\text { Se asume que los científicos } \\
\text { realizan observaciones mediadas } \\
\text { por las teorías existentes, sus } \\
\text { suposiciones personales, sus valores } \\
\text { y agendas de investigación, que } \\
\text { pueden influir en las exploraciones } \\
\text { científicas que realizan. }\end{array}$ \\
\hline
\end{tabular}


Tabla 1. Continuación

\begin{tabular}{|c|c|c|c|}
\hline & $\begin{array}{l}\text { Visiones sobre la } \\
\text { Naturaleza de la } \\
\text { Ciencia }\end{array}$ & Perspectiva ingenua & Perspectiva adecuada \\
\hline \multirow[t]{2}{*}{$\begin{array}{l}\text { Aspectos no } \\
\text { epistémicos }\end{array}$} & Impacto cultural & $\begin{array}{l}\text { El conocimiento y las formas de } \\
\text { desarrollarlo son universales: en } \\
\text { todas las culturas los científicos se } \\
\text { aproximan al estudio del mundo } \\
\text { natural desde una perspectiva } \\
\text { única, sin que existan distinciones } \\
\text { entre las diversas culturas. La } \\
\text { selección de los focos de estudio } \\
\text { tiene relevancia para la propia } \\
\text { disciplina, pero no está articulada } \\
\text { con las demandas sociales y } \\
\text { culturales de su tiempo, ni con sus } \\
\text { posibles aplicaciones. }\end{array}$ & $\begin{array}{l}\text { La ciencia es culturalmente } \\
\text { dependiente: si bien la ciencia } \\
\text { predominante es occidental, existen } \\
\text { otras formas de obtención de } \\
\text { conocimiento de la naturaleza en } \\
\text { diferentes culturas que han } \\
\text { contribuido también al desarrollo } \\
\text { del conocimiento científico, y que } \\
\text { responden a sus diversas } \\
\text { cosmovisiones. La selección de los } \\
\text { focos de estudio está fuertemente } \\
\text { influenciada por las demandas } \\
\text { sociales, culturales, políticas y } \\
\text { económicas contemporáneas }\end{array}$ \\
\hline & $\begin{array}{l}\text { Rol de } \\
\text { negociación } \\
\text { social }\end{array}$ & $\begin{array}{l}\text { Se visualiza el desarrollo del } \\
\text { conocimiento científico como un } \\
\text { proceso de exploración individual } \\
\text { conducido por el científico. El } \\
\text { nuevo conocimiento no requiere } \\
\text { validación porque responde a una } \\
\text { interpretación objetiva de los } \\
\text { datos, aplicando procedimientos } \\
\text { lógicos estandarizados y rígidos } \\
\text { que aseguran su confiabilidad } \\
\text { (método científico). }\end{array}$ & $\begin{array}{l}\text { Se visualiza el desarrollo del } \\
\text { conocimiento científico como un } \\
\text { proceso de trabajo colaborativo, } \\
\text { basado en la comunicación y el } \\
\text { logro de consensos. El nuevo } \\
\text { conocimiento científico adquiere } \\
\text { credibilidad a través de su } \\
\text { aceptación en las comunidades } \\
\text { científicas, que han acordado un } \\
\text { conjunto aceptable de estándares } \\
\text { para la evaluación de los hallazgos } \\
\text { científicos y de su confiabilidad. }\end{array}$ \\
\hline
\end{tabular}

\section{Incorporación de los aspectos de la Naturaleza de la Ciencia a la Educación Científica}

Aun cuando los aspectos presentados en el apartado anterior recogen planteamientos comunes provenientes de la literatura, su incorporación a las clases de ciencias a nivel escolar es todavía limitada. Esto se debe a obstáculos que dificultan la implementación de la $\mathrm{NdC}$ en la actividad científica escolar como: $\imath$ ) falta de tiempo, ii) escasa presencia de la Naturaleza de la Ciencia en el curriculum prescrito y en las evaluaciones externas, y iii) desconocimiento de las finalidades, aspectos esenciales, enfoques didácticos y estrategias evaluativas asociadas al trabajo explícito y didácticamente intencionado de aspectos de $\mathrm{NdC}$ por parte del profesor (Acevedo 2009).

Como resultado, numerosas investigaciones han mostrado que los estudiantes siguen manteniendo visiones ingenuas sobre la $\mathrm{NdC}$. Sin embargo, es posible hacer distinciones en los resultados encontrados, ya que se han constatado diferencias significativas según el origen cultural (Sutherland y Denick 2002), socioeconómico (Dogan y Abd-El-Khalick 2008) y la experiencia escolar de los estudiantes (García-Carmona et al. 2012). Estas diferencias han evidenciado la necesidad de hacer estudios más específicos del desarrollo de las comprensiones de los estudiantes sobre la $\mathrm{NdC}$ en contextos particulares, avanzando hacia una mejor comprensión del proceso de configuración de las visiones de los estudiantes pertenecientes a determinados contextos, según sus experiencias escolares (Dagher y Erduran 2016). 
En cuanto a la manera en que la experiencia escolar condiciona las visiones de los estudiantes, se ha evaluado la contribución a la comprensión de la $\mathrm{NdC}$ de varios contextos o escenarios escolares (García-Carmona et al. 2012). De todos ellos, la incorporación de la Historia y la Filosofía de la Ciencia ha sido uno de los recursos más intensamente sugeridos para tratar los temas de la $\mathrm{NdC}$, al mostrar cómo se construyen los conocimientos científicos en conexión con su contexto histórico y social, y se visibilizan las controversias como escenario esencial de la práctica científica (Hodson 2008; Acevedo-Díaz et al. 2017; Chamizo y García-Cruz 2020).

\section{Contribución de la Historia a la comprensión de los aspectos epistémicos y no epistémicos de la Naturaleza de la Ciencia}

Si bien se asume el potencial didáctico de la incorporación de la Historia de la Ciencia, esto es posible en la medida en que la aproximación histórica permita ejemplificar el desarrollo de las teorías científicas, conocer las relaciones entre ciencia y sociedad, e ilustrar el carácter universal y a la vez multicultural de la ciencia, planteando de manera explícita a los estudiantes los aspectos de la NdC y reflexionando sobre ellos (Acevedo-Díaz y García-Carmona 2015). Además, en el proceso de adaptar al nivel escolar episodios históricos de la ciencia, existe el riesgo de presentar imágenes deformadas (Forato, Martins y Pietrocolo 2012) que transmitan una falsa visión acumulativa y lineal de la ciencia en un proceso continuo hasta su estado actual (Monk y Osborne 1997).

Entre las diversas formas de abordar la Historia de la Ciencia reconocidas en la literatura (Izquierdo et al. 2016; Acevedo-Díaz et al. 2017), esta investigación se ha focalizado en los aportes derivados de la perspectiva biográfica, considerando que permite identificar las relaciones vinculantes entre los aspectos disciplinares, culturales, sociales, económicos, valóricos e incluso religiosos, inherentes a los contextos en los que se genera el conocimiento (Cuellar et al. 2013). Esto requiere superar las tradicionales biografías de los «grandes héroes de la ciencia», avanzando hacia la contextualización de las biografías entendiendo la ciencia como una práctica académica (Chamizo y García-Cruz 2020), mostrando los aspectos personales y sociales del trabajo científico. Integrar textos originales de los autores y visibilizar las controversias históricas en la evolución de determinados conceptos (Solbes y Traver 2001), permite resguardar el carácter subjetivo, tentativo y contextualizado de la actividad científica (Erduran \& Dagher 2014), haciendo visibles los aspectos epistémicos y no epistémicos de la $\mathrm{NdC}$ en la actividad científica escolar (Acevedo-Díaz y García-Carmona 2015).

Incorporando estos aspectos históricos, el profesorado puede dar una imagen más completa de la actividad científica, sin reducirla a sus productos, instrumentos y métodos, lo que favorecería actitudes positivas hacia la ciencia y su aprendizaje, y contribuiría al aprendizaje de ideas clave de la ciencia (Solbes y Traver 2001). Sin embargo, en el contexto latinoamericano, en el que este trabajo se desarrolló, no hemos encontrado trabajos que presten atención a las visiones sobre $\mathrm{NdC}$ de estudiantes en cuyas clases de ciencias se ha incorporado la Historia de la Ciencia desde una perspectiva biográfica, posiblemente debido a la escasez de profesores de ciencias en el sistema escolar que incorporen aspectos históricos en sus secuencias de enseñanza y aprendizaje (Cofré et al. 2019).

\section{Pregunta de investigación}

Considerando la mediación cultural en las comprensiones de los estudiantes sobre la NdC, y la identificación de la perspectiva biográfica como una estrategia que ha mostrado potencial en promover estas comprensiones, es que justificamos la relevancia de explorar en nuestro contexto particular de qué manera se configuran las visiones de estudiantes en cuya experiencia escolar se incluyen estos aspectos. Una mejor comprensión de las visiones sobre $\mathrm{NdC}$ de los estudiantes contribuiría a orientar el diseño e implementación de secuencias 
didácticas por parte de los profesores que pretendan abordar de forma explícita sus aspectos epistémicos y no epistémicos, y podría orientar a su vez los procesos de formación inicial y continua de profesores de ciencias.

Así entonces, la pregunta de investigación que orienta este trabajo es: ¿De qué manera se configuran las visiones sobre $\mathrm{NdC}$ de estudiantes chilenos en cuya experiencia escolar se incorporan aspectos históricos de la ciencia desde una perspectiva biográfica?

\section{Marco metodológico}

Dado que nuestro objetivo es caracterizar las visiones sobre la $\mathrm{NdC}$ de estudiantes chilenos en cuya experiencia escolar se incorporan aspectos históricos de la ciencia desde una perspectiva biográfica, nuestra investigación se enmarca en el paradigma cualitativo interpretativo con un enfoque de estudio de caso exploratorio (Yin 2003). Se trata de un estudio de caso exploratorio, ya que facilita la exploración de un fenómeno en su contexto, con el propósito de alcanzar abstracciones concretas y particulares a la muestra analizada, a partir de la cual puedan identificarse patrones y tendencias (Baxter y Jack 2008). Nuestros casos son cursos de educación secundaria de distintos establecimientos educacionales chilenos, considerando que todos ellos pertenecen al mismo contexto sociocultural y nivel socioeconómico, y han vivido experiencias escolares similares, dado que en todos los casos las profesoras han incorporado a la clase de ciencias aspectos históricos desde una perspectiva biográfica.

\section{Contexto y participantes}

En esta investigación se trabajó con profesores de Química de la Región del Biobío (Chile), que se desempeñan en educación secundaria, quienes participaron voluntariamente en el proyecto de investigación en el que se enmarca este trabajo, orientado a la consolidación de una comunidad de aprendizaje formada por profesores, formadores de profesores e investigadores. De los 10 profesores participantes, 4 profesoras presentaban visiones sobre $\mathrm{NdC}$ coherentes, y habían incorporado la Historia de la Ciencia en sus clases al momento de realizar este trabajo. Las cuatro profesoras empezaron a incorporar las biografías como estrategia didáctica en sus clases de ciencias, lo que contribuyó potencialmente a configurar las visiones sobre $\mathrm{NdC}$ de sus estudiantes al mostrar explícitamente la actividad científica como una práctica académica (García-Carmona et al. 2012, Chamizo y García-Cruz 2020).

De acuerdo con el reporte de las propias profesoras y sus estudiantes, la experiencia escolar combinó dos tipos de clase complementarias. La clase más frecuente consideraba como metas de aprendizaje principales conocer conceptos o teorías científicas a través de exposiciones de las profesoras que los estudiantes consideraban «didácticas», y su aplicación a ejercicios, que generalmente realizaron en forma grupal. El otro tipo de clase, menos frecuente, correspondería a una clase a través de la Historia de la Ciencia (García-Carmona et al. 2012). En algunos temas se construyeron líneas de tiempo que mostraban los hechos más importantes de la ciencia, y otros hechos contemporáneos, con un carácter evolutivo, lo que permitió al estudiantado conocer algunos personajes científicos vinculados a las ideas o teorías involucradas. La incorporación de las biografías estuvo centrada en relacionar la vida personal del científico con su formación científica y sus principales contribuciones.

Estas profesoras, y un grupo de estudiantes por cada una, constituyen entonces la muestra por conveniencia de este estudio (Creswell 2008). En total participaron 4 profesoras y 107 estudiantes, con edades comprendidas entre los 14 y los 18 años. Todos ellos fueron informados del proyecto de investigación y consintieron en que los datos derivados de su participación fueran analizados para este trabajo. 


\section{Recogida de datos}

Para la caracterización de las visiones sobre $\mathrm{NdC}$ de los estudiantes, consideramos los cinco componentes epistémicos y no epistémicos clave desarrollados en el marco teórico (ver Tabla 1): la naturaleza tentativa del conocimiento científico, la creatividad, la mediación de la teoría, el impacto cultural y el rol de la negociación social (Tsai y Liu 2005). Para poder triangular los datos entre las representaciones personales y sociales de los estudiantes, se diseñaron y aplicaron dos instrumentos para la recogida de datos: cuestionario y grupo focal. Esta decisión respondió a la necesidad de profundizar en las respuestas que los sujetos de estudio dan a los cuestionarios típicamente aplicados para la caracterización de las visiones sobre $\mathrm{NdC}$ (Lederman, Abd-El-Khalick, Bell y Schwartz, 2002). Además, considerando la construcción social de las visiones sobre la ciencia, se optó por una aproximación grupal donde emergieran visiones contrapuestas, y por tanto con mayor potencial para promover géneros discursivos más complejos que las entrevistas (Fauskanger y Mosvold 2013). En la Figura 1 representamos las etapas metodológicas, que se describen a continuación.

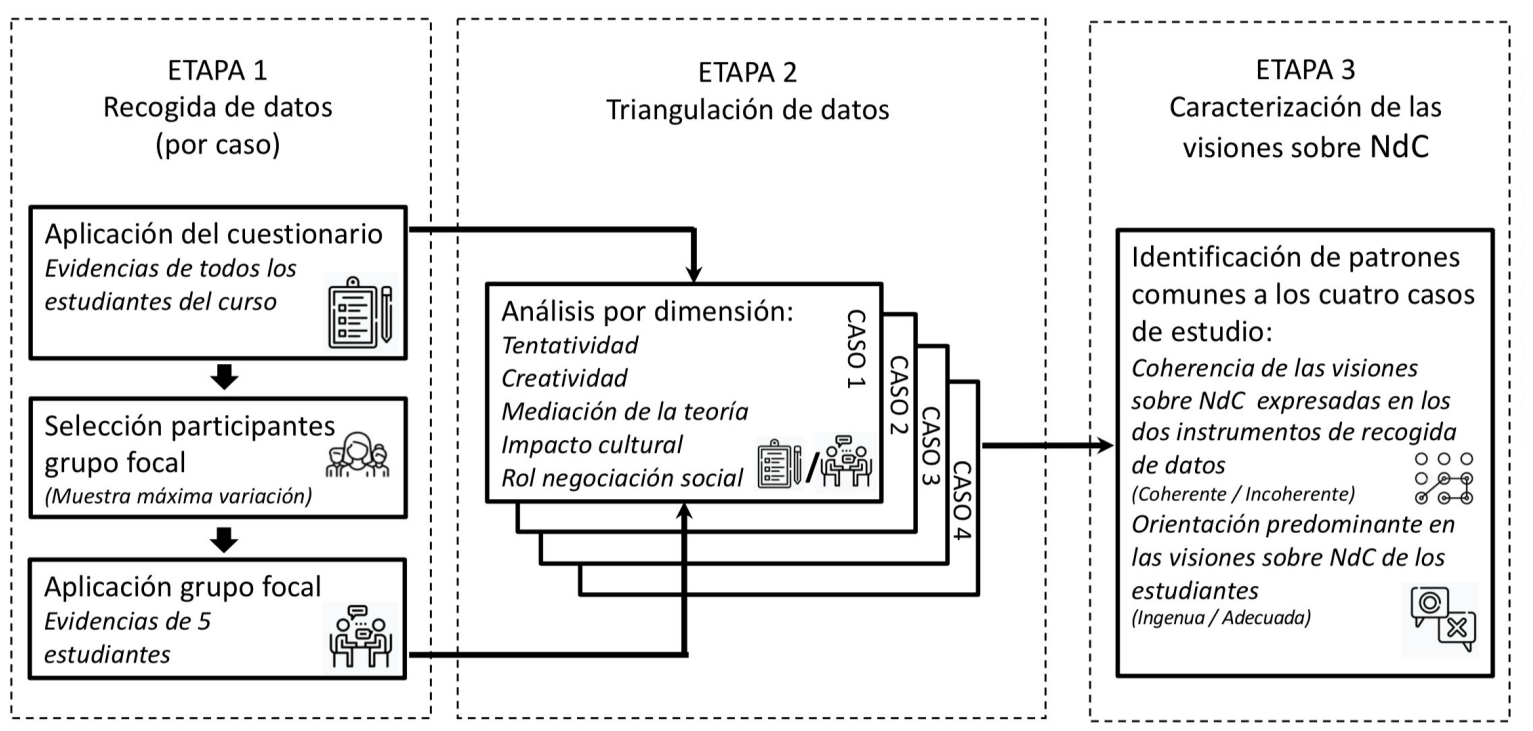

Figura 1. Sistematización de las etapas metodológicas para la caracterización de las visiones sobre la $\mathrm{NdC}$ del estudiantado de secundaria

\section{a. Representaciones personales (Cuestionario)}

El cuestionario ha sido el instrumento más comúnmente utilizado para caracterizar las visiones personales sobre $\mathrm{NdC}$ de estudiantes escolares y universitarios (García-Carmona et al. 2012). Siguiendo el enfoque metodológico de la mayoría de las propuestas revisadas, se aplicó un cuestionario de apreciación de escala Likert. Para ello se definió, para cada una de las dimensiones, una afirmación respecto de la cual los estudiantes explicitaron su grado de acuerdo o desacuerdo. Estas afirmaciones fueron seleccionadas de cuestionarios previamente aplicados a profesores en formación y en ejercicio, que ya habían sido validados (Cuellar et al. 2010).

El instrumento para la recogida de datos es entonces un cuestionario que consta de las afirmaciones que se recogen en la tabla 2, y cuya respuesta permite identificar la visión sobre $\mathrm{NdC}$ de los estudiantes según se indica. 
Tabla 2. Afirmaciones del cuestionario y relación de las respuestas con las perspectivas sobre la naturaleza de la ciencia ingenua y adecuada.

\begin{tabular}{|l|l|l|l|}
\hline $\begin{array}{l}\text { Componente de las } \\
\text { visiones } \\
\text { epistemológicas }\end{array}$ & Afirmación del cuestionario & De acuerdo & En desacuerdo \\
\hline Tentatividad (NCTC) & $\begin{array}{l}\text { 1. El desarrollo de la ciencia a lo largo } \\
\text { de la historia ha sido un proceso } \\
\text { acumulativo de más y más } \\
\text { conocimiento. }\end{array}$ & Ingenua & Adecuada \\
\hline Creatividad (NCC) & $\begin{array}{l}\text { 2. La ciencia trabaja en base a métodos } \\
\text { sistemáticamente rigurosos en los que } \\
\text { tiene poca cabida la creatividad y el } \\
\text { azar. }\end{array}$ & Ingenua & Adecuada \\
\hline $\begin{array}{l}\text { Mediación de la teoría } \\
\text { (ECT) }\end{array}$ & $\begin{array}{l}\text { 3. Los conceptos o teorías científicas } \\
\text { son la evidencia de la interpretación de } \\
\text { los científicos respecto a los } \\
\text { fenómenos. }\end{array}$ & Adecuada & Ingenua \\
\hline $\begin{array}{l}\text { Impacto cultural (IC) } \\
\text { social (RNS) }\end{array}$ & $\begin{array}{l}\text { 4. Los conceptos o teorías científicas } \\
\text { surgen del estudio de los fenómenos } \\
\text { cotidianos del entorno. }\end{array}$ & Ingenua & Adecuada \\
\hline $\begin{array}{l}\text { Rol la negociación } \\
\text { verdadero, confiable e incuestionable. }\end{array}$ & Ingenua & Adecuada \\
\hline
\end{tabular}

\section{b. Representaciones sociales (grupo focal)}

Esta estrategia de recolección de datos se aplicó de forma complementaria, para triangular con las respuestas obtenidas en el cuestionario profundizando en la caracterización de las visiones sobre $\mathrm{NdC}$ de los estudiantes. Considerando que los estudiantes del curso han compartido la experiencia escolar, se optó por aplicar un instrumento de recogida de datos que permite obtener datos mediante la interacción del grupo sobre un tópico determinado (Morgan 1996: 130). En este caso, los grupos focales se centran en temáticas previamente planteadas por el investigador, buscando, a través de la confrontación discursiva de sus miembros, las construcciones sociales que tienen sobre el tema objeto de discusión (Bolivar, Fernández y Molina 2005). Para ello se diseñó una pauta para el grupo focal que incluyó preguntas referidas a los cinco componentes de sus visiones sobre la $\mathrm{NdC}$.

En los grupos focales participaron 5 estudiantes de cada uno de los cursos, más el moderador. Para la selección de estudiantes, se utilizaron las respuestas obtenidas al cuestionario para seleccionar estudiantes con la máxima diversidad en la orientación de los cinco componentes de las visiones sobre $\mathrm{NdC}$, favoreciendo la confrontación discursiva. De esta forma los cinco estudiantes que intervienen reproducen la heterogeneidad en el grupo en cuanto a las respuestas al cuestionario, pero en un número que posibilita la localización y caracterización de los aspectos relevantes para esta investigación (Alonso 1996; Bolívar, Fernández y Molina 2005). Con ellos estimamos que puede lograrse un nivel adecuado de saturación, en el sentido de que no aparecerían elementos sustantivamente nuevos al aumentar el número de participantes. Durante el desarrollo de los grupos de discusión, éstos se registraron en audio y posteriormente se transcribieron, codificando las intervenciones del moderador como $I$, y las 
de los estudiantes como E1, E2, E3, E4 y E5, para resguardar la confidencialidad de los participantes.

\section{Análisis de los datos}

El análisis de los datos constó de dos niveles: un primer nivel de análisis en que se sistematizaron las respuestas de los estudiantes de cada uno de los cursos al cuestionario, y posteriormente se triangularon con las respuestas obtenidas en el grupo focal. Esto posibilitó identificar la orientación sobre $\mathrm{NdC}$ predominante en cada uno de los casos, identificando la coherencia entre las respuestas al cuestionario y el grupo focal.

En el análisis se aplicaron los criterios definidos en la tabla 2 para identificar, respecto a cada estudiante, la orientación ingenua o adecuada en cada uno de los componentes de sus visiones sobre NdC. Posteriormente se realizó un análisis del contenido del discurso, identificando los fragmentos de la discusión que se referían a las visiones sobre $\mathrm{NdC}$, y categorizando las intervenciones de cada estudiante de acuerdo con el componente al que se refiere, y su orientación (Tabla 1). A continuación, se representó en los cuatro casos, para cada uno de los componentes, el porcentaje de estudiantes que presentaban visiones ingenuas y adecuadas tanto en el cuestionario como en el grupo focal; lo que permitió visualizar las orientaciones sobre $\mathrm{NdC}$ a nivel grupal, así como comparar los datos obtenidos mediante las dos estrategias de recogida de datos para la triangulación.

En el segundo nivel de análisis se identificaron patrones comunes a los cuatro casos de estudio, caracterizando las visiones sobre $\mathrm{NdC}$ del estudiantado de secundaria participante cuyas clases de ciencias incorporaron las bibliografías científicas.

\section{Resultados}

De acuerdo con el enfoque metodológico de estudio de caso exploratorio de esta investigación, los resultados se presentan para cada caso, organizados en los cinco componentes de las visiones sobre $\mathrm{NdC}$ (Tsai y Liu, 2005) y en los dos instrumentos de recogida de datos utilizados: cuestionario y grupo focal. En los casos en que aparecen incoherencias entre las respuestas a los dos instrumentos, se presentan extractos de los grupos focales que permiten discutir con mayor profundidad en torno a las visiones de los estudiantes en el componente.

\section{Caso 1}

El caso 1 corresponde a un curso de I Medio (13-14 años) con 37 estudiantes. En la figura 2 se presenta el consolidado del análisis de sus respuestas al cuestionario y al grupo focal.

En cuanto a la naturaleza creativa de la ciencia, la mediación de la teoría y la tentatividad del conocimiento científico, los estudiantes presentan visiones predominantemente adecuadas tanto en las respuestas en el cuestionario como en el grupo focal, reconociendo el rol de los científicos en la interpretación de los datos obtenidos experimentalmente, mediadas por las teorías existentes, y el carácter dinámico del conocimiento científico que se genera (Tsai y Liu 2005; Vázquez y Manassero 1999). En ese sentido, los estudiantes presentan visiones coherentes en torno a los aspectos epistémicos de la NdC (Acevedo-Díaz y García-Carmona 2016). 


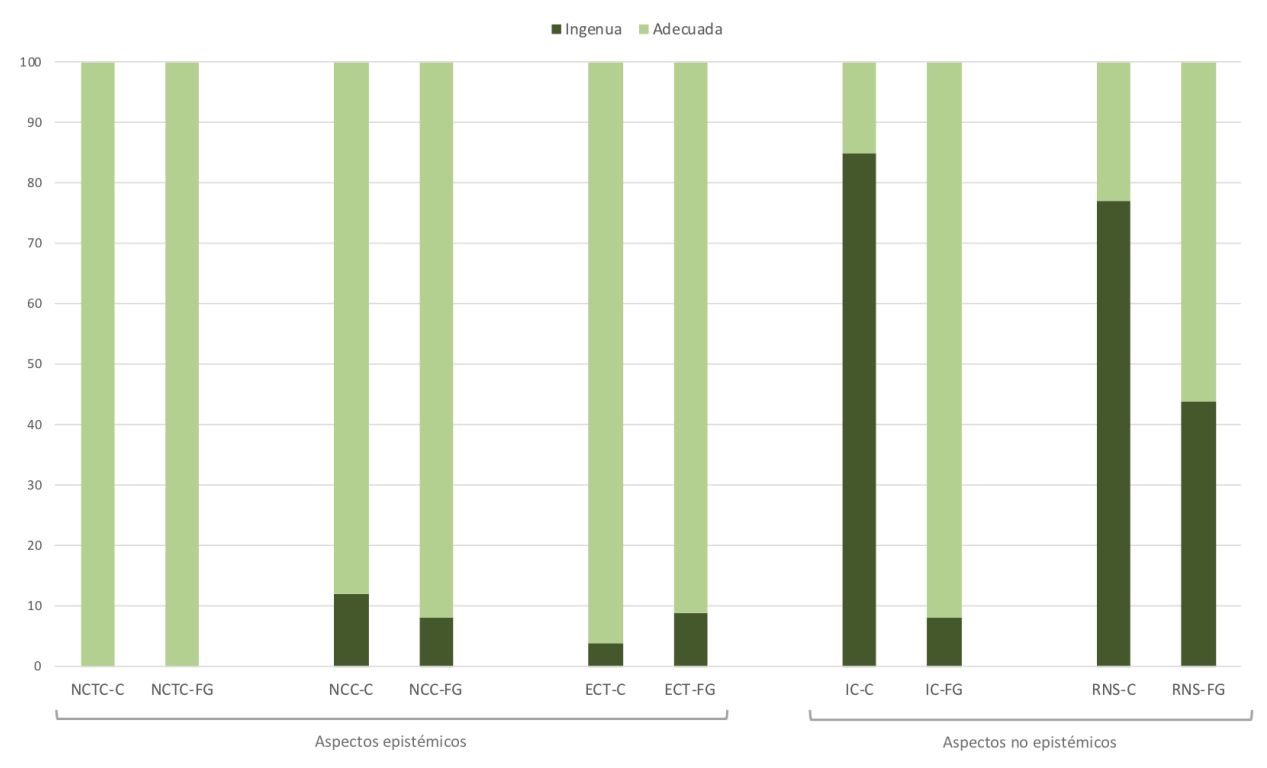

Figura 2. Porcentaje de estudiantes con orientación ingenua o adecuada, en los cinco componentes de las visiones sobre la NdC (Tsai y Liu 2005), en el cuestionario y grupo focal, para el caso 1.

En cuanto al rol de la negociación social, encontramos una orientación ingenua en el cuestionario, asumiendo el trabajo científico como verdadero, confiable e incuestionable. Sin embargo, en el grupo focal, esta tendencia disminuye dando espacio a algunas reflexiones en torno a la necesidad de validación del conocimiento, reconociendo que históricamente el aporte de algunos científicos no fue tenido en cuenta, tal y como puede verse en este fragmento de la discusión:

I: Esta visión de la ciencia no estructurada, cles cambiaría en algo la imagen del científico? E1:E2:E4:E5: Sí.

I: ¿Cómo?

E3: Nos armaríamos una idea de que los científicos no son tan apartados.

E1: Que tuvieron vida...

E4: Porque hay algunos que los desterraron del lugar donde vivían porque los creían locos, entonces eso es más entender como ellos sentían. Para después recordar que la ciencia puede haber sido muy importante pero también en su momento no la tomaron en cuenta, no la supieron valorar.

Ocurre lo mismo en el caso del impacto cultural, donde encontramos que los estudiantes presentan una marcada tendencia ingenua en el cuestionario, pero en el grupo focal aparecen posturas mayoritariamente adecuadas que reconocen la mediación cultural y social en la actividad científica.

Para los componentes rol de la negociación social e impacto cultural, encontramos visiones expresadas en el cuestionario y el grupo focal poco coherentes, en relación con los aspectos no epistémicos de la NdC (Acevedo-Díaz y García-Carmona 2016), dado que los estudiantes sitúan el trabajo de los científicos en una dimensión personal más que social. Al explorar con mayor detalle las intervenciones en el grupo focal que se refieren a estos componentes con una orientación adecuada, encontramos que en todos los casos están ancladas en ejemplos particulares que parecen resultar anecdóticos, más que ejemplos representativos de la actividad científica como una práctica académica (Chamizo y García Cruz 2020). 


\section{Caso 2}

El caso 2 corresponde a un curso de I Medio (13-14 años) con 26 estudiantes. En la figura 3 se presenta el consolidado del análisis de sus respuestas al cuestionario y al grupo focal.

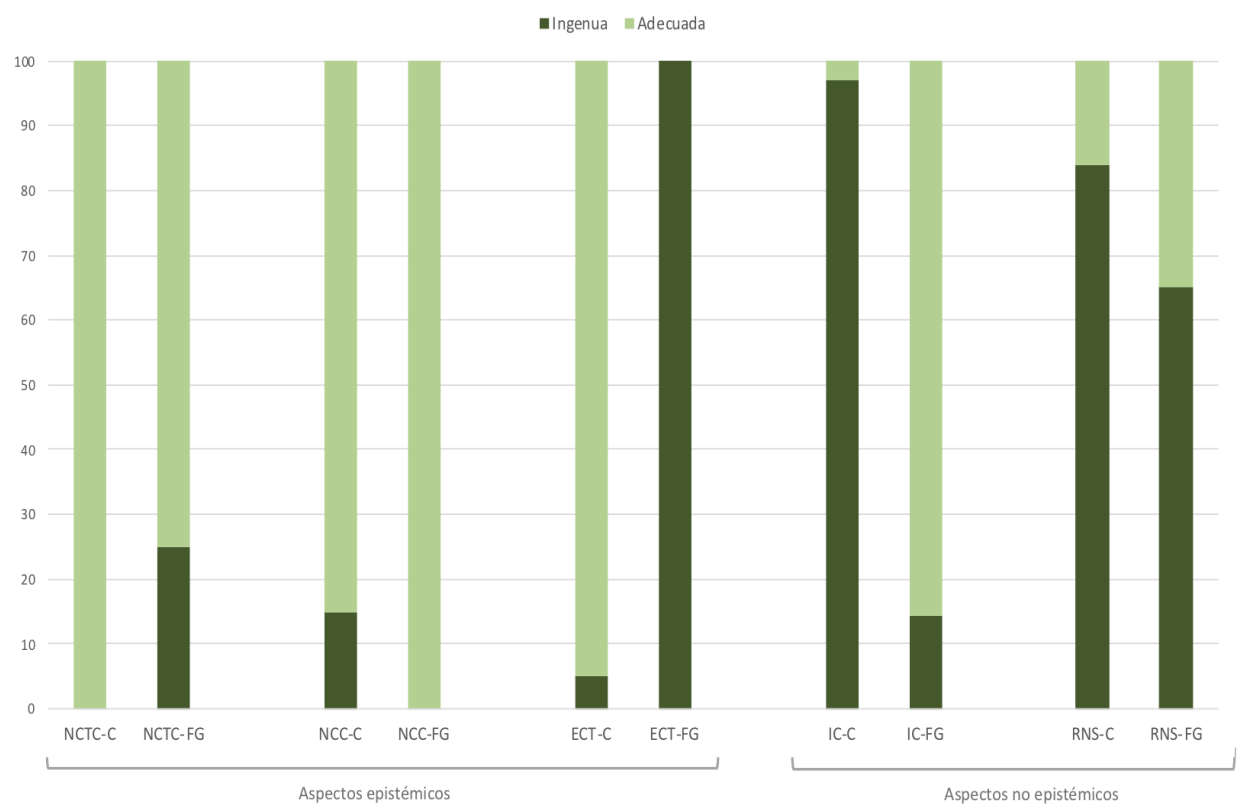

Figura 3. Porcentaje de estudiantes con orientación ingenua o adecuada, en los cinco componentes de las visiones sobre la NdC (Tsai y Liu 2005), en el cuestionario y grupo focal, para el caso 2.

En cuanto a la naturaleza creativa de la ciencia y la tentatividad del conocimiento científico, los estudiantes presentan visiones adecuadas tanto en las respuestas al cuestionario como en el grupo focal, con concepciones sobre la actividad científica ligada a la rigurosidad de los procesos, en la que puede estar presente la creatividad y el azar, y confiriéndole un carácter dinámico (Tsai y Liu 2005).

En relación con la mediación de la teoría, se identifica una visión adecuada en el cuestionario, donde se otorga importancia a la subjetividad del científico y a su interpretación en los hechos estudiados. En el grupo focal la tendencia cambia hacia posturas ingenuas, aunque este componente aparece solamente en una oportunidad a lo largo de toda la discusión, lo que impide poder profundizar en la incoherencia que aparece.

Respecto del impacto cultural se identifica una marcada tendencia ingenua en el cuestionario, contraria a las intervenciones en el grupo focal, donde aparecen posturas predominantemente adecuadas. En el grupo focal los estudiantes plantean que si bien las teorías científicas surgen de la observación de fenómenos cotidianos, en algunos casos están fuertemente influenciadas por factores externos, tal como puede verse en este fragmento:

I: ¿y ese algo [las teorías científicas] dónde está?

E2: en lo cotidiano

E4: en los fenómenos que suceden, y ahí empiezan a buscar de que se trata.

I: Y, ¿tú crees que eso [el desarrollo del conocimiento científico] fue fácil para ellos?

$\mathrm{E} 2: \mathrm{E} 4: \mathrm{E} 5$ :no

E3: yo creo que fue difícil, porque hay algunos hechos científicos, el caso cuando predominaba la religión católica, algunos hechos de la ciencia que contradecían eso y había mucha

persecución hacia los científicos y muchos fueron encerrados. 
Respecto al rol de la negociación social, se identifica una marcada visión ingenua, tanto en el cuestionario como el en grupo focal, caracterizando el conocimiento científico como algo confiable e incuestionable, cuya finalidad es la comprensión de los hechos del mundo.

Para los componentes asociados a los aspectos no epistémicos de las visiones sobre $\mathrm{NdC}$ (Acevedo-Díaz y García-Carmona 2016) encontramos visiones ingenuas en torno al rol de la negociación social y adecuadas aunque incoherentes para el impacto Cultural, lo que sitúa el trabajo de los científicos en una dimensión personal, desconociendo la actividad científica como una práctica académica mediada social y culturalmente (Chamizo y García-Cruz 2020), salvo en algunos casos particulares.

\section{Caso 3}

El caso 3 corresponde a un curso de III Medio (16-17 años) con 21 estudiantes. En la figura 4 se presenta el consolidado del análisis de sus respuestas al cuestionario y al grupo focal.

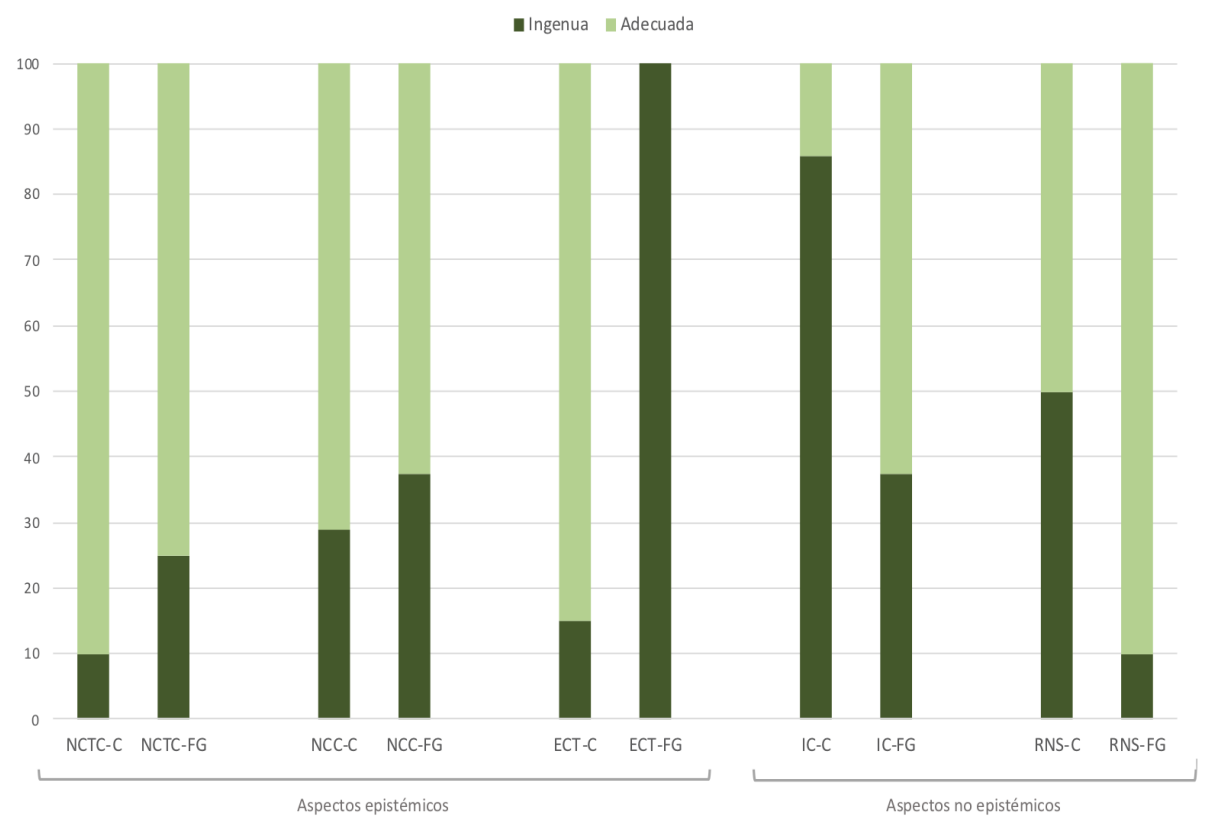

Figura 4. Porcentaje de estudiantes con orientación ingenua o adecuada, en los cinco componentes de las visiones sobre la NdC (Tsai y Liu 2005), en el cuestionario y grupo focal, para el caso 3.

Respecto a la naturaleza creativa y la tentatividad del conocimiento cientifico, las visiones de los estudiantes son mayoritariamente adecuadas, tanto en el cuestionario como en el grupo focal. El grupo de estudiantes reconoce la rigurosidad del trabajo científico en la construcción del conocimiento, que no necesariamente excluye la posibilidad de la presencia del azar y la creatividad, y que éste surge por la capacidad de los científicos para hacerse preguntas sobre los fenómenos observados en el entorno, considerando el carácter subjetivo y tentativo de la ciencia (Dhager y Erduran 2016).

En relación a la mediación de la teoría se identifica una visión predominante adecuada en el cuestionario, en la que se cuestiona la existencia de una realidad científica externa al sujeto que construye ciencia. En el grupo focal la tendencia cambia hacia posturas ingenuas, pero al igual que en el caso II, este componente aparece solamente en la intervención de una estudiante en el grupo focal, lo que nuevamente impide poder profundizar en esta incoherencia.

En relación al impacto cultural, se identifica una marcada tendencia ingenua en el cuestionario, cuestionando la aplicabilidad de la ciencia, que da paso a posturas ingenuas y adecuadas coexistentes en el grupo focal, tal como puede verse en las intervenciones de los estudiantes: 
I: ¿Cómo se imaginan que se genera el conocimiento científico? ¿Cómo creen que piensan los científicos?

E3: Primero tienen una duda o una pregunta y de ahí salen las investigaciones y para eso ocupan el método científico.

E2: La visión que tengo de un científico, no es del típico con delantal y que está en un laboratorio, sino que como, bueno, la ciencia no partió en un laboratorio sino por cosas que pasaron en el entorno entonces. Me imagino una persona común y corriente yendo a ver cómo es la naturaleza y ver por qué sopla el viento, por poner un ejemplo.

E4: Según yo la ciencia surge por la curiosidad de algunas personas por saber.

E1: Lo que sucede en nuestro entorno.

El componente rol de la negociación social de la ciencia, en este caso, muestra en el cuestionario una inicial polarización entre las dos visiones, ingenua y adecuada, en la que se evidencian puntos de vista opuestos en cuanto a la aceptación de la universalidad, confiabilidad y dogmatismo del conocimiento científico. Por su parte, este componente es mayoritariamente adecuado en el grupo focal, en donde se reconoce que el conocimiento científico se genera mediante un trabajo colaborativo (Tsai y Liu 2005).

Al igual que en los casos anteriores, los estudiantes no parecen tener una visión consolidada en cuanto a los aspectos no epistémicos de la NdC (Acevedo-Díaz y García-Carmona 2016), y si bien identifican ciertas influencias del entorno en los intereses y motivaciones de los científicos, se refieren a estas influencias de forma vaga.

\section{Caso 4}

El caso 4 corresponde a un curso de IV Medio (17-18 años) con 26 estudiantes. En la figura 5 se presenta el consolidado del análisis de sus respuestas al cuestionario y al grupo focal.

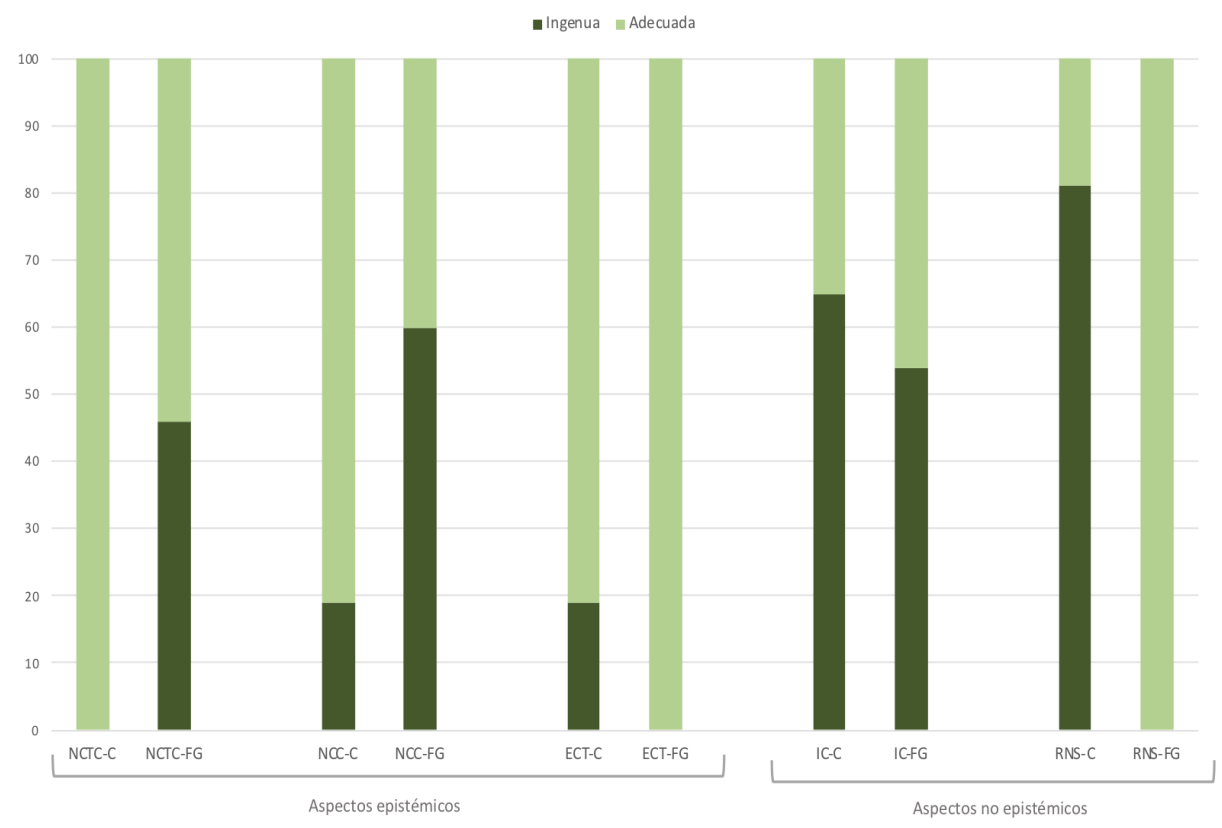

Figura 5. Porcentaje de estudiantes con orientación ingenua o adecuada, en los cinco componentes de las visiones sobre la NdC (Tsai y Liu 2005), en el cuestionario y grupo focal, para el caso 4.

En cuanto al rol de la negociación social y el impacto cultural, los estudiantes presentan visiones predominantemente ingenuas en las respuestas al cuestionario. En el caso del impacto cultural, esta tendencia se mantiene en el grupo focal en el que las intervenciones de los estudiantes muestran la escasa relevancia que le otorgan a las demandas sociales y culturales en la práctica científica. Sin embargo, en el caso del rol de la negociación social, las discusiones del grupo focal 
muestran una orientación adecuada en la que se reconoce la necesidad de validación del conocimiento, lo que es incoherente con las respuestas al cuestionario en que consideraban el conocimiento científico como incuestionable (Tsai y Liu 2005; Vázquez y Manassero 1999).

En cuanto a la naturaleza creativa del conocimiento científico, la mediación de la teoría y la tentatividad del conocimiento cientifico los estudiantes presentan visiones predominantemente adecuadas en el cuestionario. En el caso de la mediación de la teoría en la actividad científica, esta tendencia se mantiene en el grupo focal. En el caso de la naturaleza creativa y tentativa del conocimiento científico, las intervenciones de los estudiantes muestran creencias ingenuas, incoherentes con sus respuestas al cuestionario, tal y como se muestra en el fragmento de discusión a continuación, referida a la tentatividad:

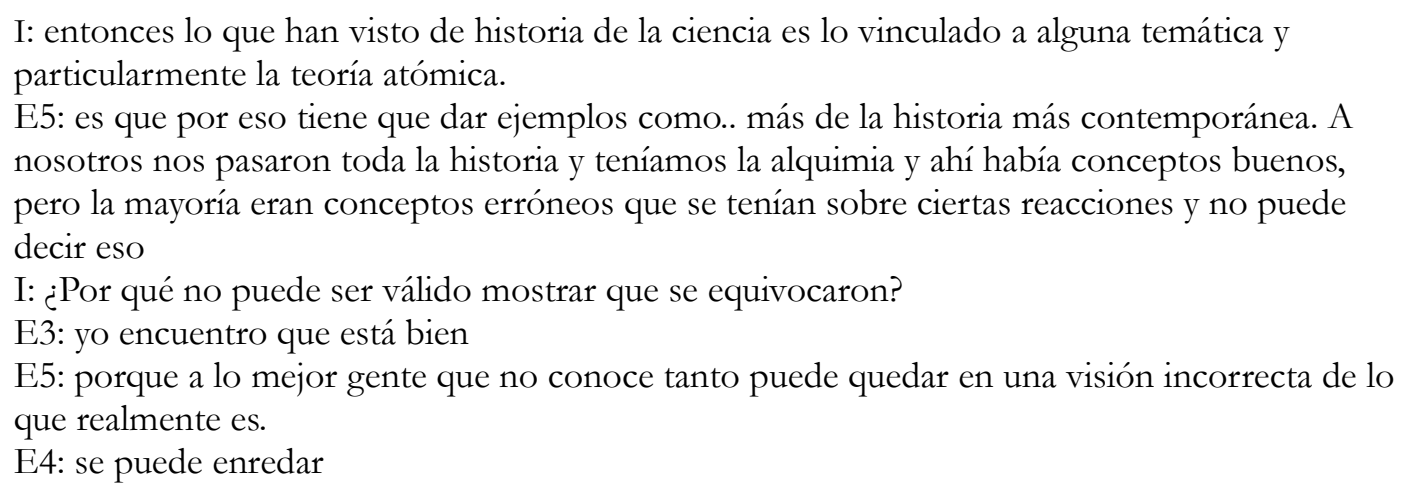

En síntesis, para los componentes de impacto cultural y mediación de la teoría encontramos visiones coherentes, ingenuas en el primer caso, y adecuadas en el segundo. Los estudiantes son consistentes en considerar que el avance de la ciencia está mediada por la teoría y sus avances responden principalmente a la relevancia del foco de estudio para la propia disciplina (Tsai y Liu 2005). En el caso del rol de la negociación social, y la naturaleza creativa y tentativa de la ciencia, las visiones son incoherentes; aun cuando los aspectos sociales de la ciencia parecieran ser reconocidos por los estudiantes, nuevamente aparecen asociados a ejemplos particulares en el grupo focal. Así entonces, las visiones adecuadas en sus aspectos epistémicos y no epistémicos de la NdC no están consolidados en este grupo (Acevedo-Díaz y García-Carmona 2016).

\section{Patrones comunes a los cuatro casos de estudio}

Los cuatro casos de estudio presentaron experiencias similares en cuanto a la incorporación de las biografías científicas en algunas temáticas curriculares, complementando las clases tradicionales a través de la construcción de líneas de tiempo que se centraron, principalmente, en dar a conocer los científicos que contribuyeron al desarrollo de ideas claves, y sus principales aportes. La forma en que se incorporaron las biografías científicas tendría entonces un bajo nivel de contextualización (Solbes y Traver 2001), y su potencial didáctico estaría limitado por las escasas oportunidades que los estudiantes tuvieron de reflexionar sobre aspectos de la NdC de forma explícita (Acevedo-Díaz y García-Carmona 2016). Además, el uso de las líneas de tiempo podría responder a una excesiva simplificación de los hechos históricos, llevando a transmitir visiones acumulativas de la ciencia (Monk y Osborne 1997).

Para facilitar la identificación de patrones comunes a los cuatro casos de estudio, en la tabla 3 se presenta el consolidado de las respuestas al cuestionario y grupo focal de los cuatro casos. Para cada uno de los componentes se registra si la orientación predominante es ingenua (I) o adecuada (A), y también, en el subíndice, la coherencia (C) o incoherencia (I) entre las respuestas del cuestionario y el grupo focal. 
Tabla 3. Consolidado de orientaciones predominantes en las visiones epistemológicas de los cuatro casos.

\begin{tabular}{|l|c|c|c|c|}
\hline COMPONENTE & CASO 1 & CASO 2 & CASO 3 & CASO 4 \\
\hline Tentatividad & $\mathrm{A}_{\mathrm{C}}$ & $\mathrm{A}_{\mathrm{C}}$ & $\mathrm{A}_{\mathrm{C}}$ & $\mathrm{A}_{\mathrm{I}}$ \\
\hline Creatividad & $\mathrm{A}_{\mathrm{C}}$ & $\mathrm{A}_{\mathrm{C}}$ & $\mathrm{A}_{\mathrm{C}}$ & $\mathrm{A}_{\mathrm{I}}$ \\
\hline Mediación de la teoría & $\mathrm{A}_{\mathrm{C}}$ & $\mathrm{I}_{\mathrm{I}}$ & $\mathrm{I}_{\mathrm{I}}$ & $\mathrm{I}_{\mathrm{C}}$ \\
\hline Impacto cultural & $\mathrm{A}_{\mathrm{I}}$ & $\mathrm{I}_{\mathrm{I}}$ & $\mathrm{I}_{\mathrm{I}}$ & $\mathrm{I}_{\mathrm{C}}$ \\
\hline Rol de la negociación social & $\mathrm{I}_{\mathrm{I}}$ & $\mathrm{I}_{\mathrm{C}}$ & $\mathrm{A}_{\mathrm{C}}$ & $\mathrm{A}_{\mathrm{I}}$ \\
\hline
\end{tabular}

Analizando conjuntamente las visiones sobre $\mathrm{NdC}$ identificadas en los cuatro casos, podemos identificar orientaciones adecuadas consolidadas en cuanto al componente creativo de la actividad científica y al carácter tentativo del conocimiento científico. Esto nos permite afirmar que la incorporación de las biografías a las clases de ciencias ha configurado en los estudiantes que participaron de este estudio visiones sobre la $\mathrm{NdC}$ que resguardan su carácter subjetivo y tentativo (Dagher y Erduran 2016), lo que corresponde a sus aspectos epistémicos (AcevedoDíaz y García-Carmona 2016).

En cuanto a la mediación de la teoría, hemos encontrado visiones adecuadas consolidadas en dos de los casos, e ingenuas e incoherentes en otros dos. En los casos en que las visiones presentaron estas incoherencias, este ha sido un componente con muy baja presencia en los grupos focales, lo que ha dificultado poder ahondar en las incoherencias que aparecen. Para ello serán necesarios estudios posteriores que profundicen en este aspecto epistémico de las visiones sobre NdC de los estudiantes (Acevedo-Díaz y García-Carmona 2016).

En los componentes de rol de la negociación social e impacto cultural, no se observa un patrón claro en las visiones de los estudiantes, ya que aparece predominio de ambas visiones, en algunos casos coherentes y en otros incoherentes. Sin embargo, dado que en ningún caso aparecen visiones ingenuas coherentes en estos dos componentes, podemos afirmar que las visiones del estudiantado, en los cuatro casos, se encuentra en transición hacia orientaciones adecuadas en cuanto al carácter contextualizado de la actividad científica, aunque estos aspectos no epistémicos todavía no se consolidan (Dagher y Erduran 2016). Este resultado se condice con el bajo nivel de contextualización que, de acuerdo con la descripción de las profesoras y estudiantes, tuvieron las biografías científicas en los cursos (Solbes y Traver 2001). Las biografías científicas, tal y como fueron abordadas, mantienen visiones sobre la actividad científica en una dimensión personal, cuya práctica se reconoce como de naturaleza académica solamente en algunos de los casos (Chamizo y García-Cruz 2020).

Finalmente, nuestros resultados confirman la existencia de visiones sobre la $\mathrm{NdC}$ eclécticas (Vázquez y Manassero 1999), dado que en ninguno de los casos hemos encontrado visiones ingenuas o adecuadas en los cinco componentes estudiados. Aun así, considerando la escasez de profesores del sistema escolar que incorporen aspectos históricos en las secuencias de enseñanza y aprendizaje (Cofré et al. 2019) y que la mayoría de los estudios previos sobre las visiones sobre la $\mathrm{NdC}$ de los estudiantes han reportado visiones ingenuas sobre la ciencia (García-Carmona et al. 2012), los resultados muestran que la incorporación de las biografías científicas contribuyó en la configuración de visiones sobre la ciencia más adecuadas.

\section{Conclusiones e implicaciones}

De acuerdo con la literatura, las clases de ciencias habituales contribuyen escasamente a la comprensión de la NdC por parte de los estudiantes (García-Carmona et al. 2012). En este trabajo hemos asumido que la incorporación de la Historia de la ciencia desde una perspectiva 
biográfica ha proporcionado a los estudiantes una experiencia escolar que ha contribuido significativamente a configurar visiones más apropiadas sobre la NdC (Hodson 2008). En el relato de los propios estudiantes se identifica que, aun cuando las profesoras presentan visiones epistemológicas constructivistas coherentes, la incorporación de la Historia de la Ciencia es todavía escasa y fuertemente dependiente del contexto disciplinar, posiblemente debido a obstáculos institucionales asociados a la escasez de tiempo y de presencia curricular (Acevedo 2009). Las biografías están centradas en establecer relaciones entre las características personales de los científicos y sus contribuciones, y establecer líneas cronológicas, y tienen por tanto un bajo nivel de contextualización (Solbes y Traver 2001).

Si bien el bajo nivel de contextualización limita el potencial didáctico de la incorporación de la Historia de la ciencia en términos de promover la configuración de visiones adecuadas sobre la NdC en los estudiantes (Chamizo y García-Cruz 2020), el análisis de los datos obtenidos a través del cuestionario y los grupos focales nos ha permitido caracterizar los componentes en los que han logrado avanzar hacia perspectivas adecuadas (Tsai y Liu 2005). Los estudiantes identifican claramente el conocimiento científico como un conjunto de representaciones de los científicos sobre el mundo natural, mediadas por la teoría y de carácter tentativo, mostrando las posibilidades que las biografías científicas proporcionan para configurar visiones sobre la $\mathrm{NdC}$ adecuadas en su dimensión epistémica (Acevedo-Díaz y GarcíaCarmona 2016).

Sin embargo, los estudiantes no logran establecer con claridad el rol de la negociación social ni el impacto cultural en el desarrollo de la ciencia, dado que solamente logran asociar estos atributos a la actividad de determinados científicos, vinculados a las biografías realizadas. Aun cuando la contextualización cultural y social de las biografías permiten incorporar aspectos no epistémicos de la NdC (Acevedo-Díaz y García-Carmona 2016), este vínculo queda restringido al caso particular de la biografía, y no se generaliza como un atributo de la actividad científica.

Nuestros resultados evidencian la necesidad de incorporar ciertos aspectos a las biografías científicas para contribuir de mejor manera a la comprensión de los estudiantes de la NdC: $\imath$ ) visibilizar las controversias históricas en el desarrollo de los conceptos, como una estrategia para superar la visión epistemológica acumulativa (Solbes y Traver 2001; Acevedo-Díaz et al. 2017); ii) incorporar contribuciones a la ciencia provenientes de otras culturas y cosmovisiones, con especial énfasis en los aportes de los pueblos originarios latinoamericanos, para evidenciar el impacto cultural en el desarrollo del conocimiento científico (Lederman 2006); y finalmente iii) lograr un mayor grado de contextualización de las biografías, incorporando aspectos sociales que permitan comprender la relación ciencia-sociedad y la forma en que media en el desarrollo del conocimiento científico en general (Solbes y Traver 2001).

Estos aspectos proporcionarían a los estudiantes una experiencia escolar con mayor potencial para transformar su visión de los componentes epistémicos y no epistémicos de la $\mathrm{NdC}$, contribuyendo a una mejor comprensión de la actividad científica. Esto favorecería, de acuerdo con la literatura, actitudes positivas de los estudiantes en relación con la ciencia y su aprendizaje (Cofré et al. 2019), el aprendizaje de las ciencias a nivel escolar y la toma de decisiones de los estudiantes sobre cuestiones socio científicas (García-Carmona et al. 2012).

Continuar indagando en la configuración de las visiones sobre la $\mathrm{NdC}$ de los estudiantes en nuestro contexto, incorporar al profesorado en comunidades de aprendizaje y explorar de qué manera estas instancias de colaboración docente permiten fortalecer las experiencias escolares en sus respectivas clases de ciencias, contribuirá a consolidar progresivamente, con base en evidencia, orientaciones didácticas para la incorporación de las biografías científicas a las clases 
de ciencias con potencial para aportar significativamente a la alfabetización científica de los estudiantes chilenos (McComas 2008).

\section{Agradecimientos}

Este trabajo deriva del proyecto Fondecyt Iniciación 11150509 titulado "La formación del profesorado de ciencias en ejercicio, orientada en el desarrollo profesional docente y las comunidades de aprendizaje, y su aporte a la calidad de las competencias científicas escolares", financiado por la Comisión Nacional de Investigación Científica y Tecnológica (CONICYT), Gobierno de Chile.

\section{Bibliografía}

Abd-El-Khalick F. (2012). Nature of science in science education: Toward a coherent framework for synergistic research and development. En B. Fraser, K. Tobin y C. J. McRobbie (Eds.), Second international bandbook of science education (pp. 1041-1060). Dordrecht: Springer.

Acevedo J.A. (2009). Conocimiento didáctico del contenido para la enseñanza de la naturaleza de la ciencia (II): una perspectiva. Revista Eureka sobre Enseñanza y Divulgación de las Ciencias, 6(2), 164-169.

Acevedo-Díaz J.A., García-Carmona A. (2016). «Algo antiguo, algo nuevo, algo prestado». Tendencias sobre la naturaleza de la ciencia en la educación científica. Revista Eureka sobre Enseñanza y Divulgación de las Ciencias, 13(1), 3-19.

Acevedo-Díaz J.A., García-Carmona A., Aragón M. (2017). Historia de la ciencia para enseñar naturaleza de la ciencia: una estrategia para la formación inicial del profesorado de ciencia. Educación quimica,28(3), 140-146.

Acevedo-Díaz J.A., García-Carmona A., Aragón-Méndez M. (2017). Enseñar y aprender sobre naturaleza de la ciencia mediante el análisis de controversias de historia de la ciencia: resultados y conclusiones de un proyecto de investigación. Madrid: OEI.

Alonso L.E. (1996). El grupo de discusión en su práctica: memoria social, intertextualidad y acción comunicativa. Revista internacional de sociología ,13, 5-36.

Baxter P., Jack S. (2008). Qualitative case study methodology: study design and implementation for novice researchers. The Qualitative Report, 13(4), 544-559.

Bolívar A., Fernández M., Molina E. (2005). Investigar la identidad profesional del profesorado: Una triangulación secuencial. In Forum Qualitative Sozialforschung/Forum Qualitative Social Research, 6 (1), Art. 12.

Chamizo J.A., García-Cruz J.C. (2020). Una experiencia en la formación de docentes a partir de la historia y filosofía de la química. Revista Eureka sobre Enseñanza y Divulgación de las Ciencias 17(1), 1601.

Cofré H., Vergara C. (2010). La formación de profesores de ciencia en Chile: desarrollo, estado actual y futuros desafíos. En Cofré, H. (Ed.) Cómo mejorar la enseñanza de las ciencias en Chile, (pp. 257-278). Santiago de Chile: Ediciones Universidad Cardenal Silva Henríquez.

Cofré H., Núñez P., Santibáñez D., Pavez J.M., Valencia M., Vergara C. (2019). A Critical Review of Students' and Teachers' Understandings of Nature of Science. Science \& Education, 28 (3-5), 1-44.

Creswell J.W. (2013). Qualitative inquiry and research design: choosing among five approaches. Lincoln: Sage. 
Cuellar L., Quintanilla M., Marzabal A. (2010). La importancia de la historia de la química en la enseñanza escolar: análisis del pensamiento y elaboración de material didáctico de profesores en formación. Ciência \& Educação, 16(2), 277-291.

Cuellar L., Quintanilla M., García A. (2013). Las Biografías científicas en el aula de clase. Una nueva propuesta que integra creatividad y aprendizaje del conocimiento científico escolar. Enseñanza de las ciencias: revista de investigación y experiencias didácticas, (Extra), 889892.

Dagher Z.R., Erduran S. (2016). Reconceptualizing nature of science for science education. Science \& Education, 25(1-2), 147-164.

Dhingra K. (2003). Thinking about television science: How students understand the nature of science from different program genres. Journal of research in science teaching, 40(2), 234256.

Dogan N., Abd-El-Khalick F. (2008). Turkish grade 10 students' and science teachers' conceptions of nature of science: A national study. Journal of Research in Science Teaching: The Official Journal of the National Association for Research in Science Teaching, 45(10), 10831112.

Duschl R.A. (1990). Restructuring science education: The importance of theories and their development. Teachers College Press.

Fauskanger J., Mosvold R. (2013). Studying teachers' epistemic beliefs by using focused discussions based on MKT items. In Proceedings of the VIII CERME. Antalya, Turquía.

Forato T.C., Martins R., Pietrocola M. (2012). History and nature of science in high school: Building up parameters to guide educational materials and strategies. Science \& Education, 21(5), 657-682.

García-Carmona A., Manassero M.A., Vázquez A. (2012). Comprensión de los estudiantes sobre naturaleza de la ciencia: análisis del estado actual de la cuestión y perspectivas. Enseñanza de las Ciencias, 30(1), 23-34.

González-Weil C., Waring M.G., Ahumada G., Cisternas A., Pérez J.L., Valenzuela J.S. (2014). Contribución del trabajo colaborativo en la reflexión docente y en la transformación de las prácticas pedagógicas de profesores deficiencia escolares y universitarios. Pensamiento Educativo. Revista de Investigación Educacional Latinoamericana, 51(2), 75-85.

Hodson D. (2008). Towards scientific literacy: A teachers' guide to the history, philosophy and sociology of science. Brill Sense.

Izquierdo M., García A., Quintanilla M., Adúriz-Bravo A. (2016). Historia, filosofía y didáctica de las ciencias: aportes para la formación del profesorado de ciencias. Bogotá: Universidad Distrital Francisco José de Caldas.

Lederman N.G. (2006) Research on nature of science: reflections on the past, anticipations of the future. Asia-Pacific Forum on Science Learning and Teaching, 7(1), 1-11.

Lederman N.G., Abd-El-Khalick F., Bell R.L., Schwartz R.S. (2002). Views of nature of science questionnaire: toward valid and meaningful assessment of learners' conceptions of nature of sciencie. Journal of research in science teaching, 39(6), 497-521.

Matthews M.R. (2017). La enseñanza de la ciencia: un enfoque desde la bistoria y la filosofía de la ciencia. Bogotá: Fondo de Cultura Economica. 
McComas W.F. (2008). Seeking historical examples to illustrate key aspects of the nature of science. Science \& Education, 17(2-3), 249-263.

Monk M., Osborne J. (1997). Placing the history and philosophy of science on the curriculum: A model for the development of pedagogy. Science education, 81(4), 405-424.

Morgan D. (1996). Focus groups. Annual Review of Sociology, 22, 129-152.

Siso Z., Sánchez I.. Cuellar L. (2019). Una experiencia de aprendizaje en formación continua de profesores de química fundamentada en naturaleza de la ciencia y tecnología. Góndola, enseñanza y aprendizaje de las ciencias, 14(2), 229-252.

Siso Z., Cuellar L. (2017). Relaciones entre las concepciones de naturaleza de la ciencia y la tecnología, y de la enseñanza y el aprendizaje de las ciencias de profesores de química en ejercicio. Una primera aproximación al esquema conceptual del profesor. Tecné, Episteme y Didaxis: TED, 41, 17-36.

Solbes J., Traver M. (2001). Resultados obtenidos introduciendo historia de la ciencia en las clases de física y química: mejora de la imagen de la ciencia y desarrollo de actitudes positivas. Enseñanza de las ciencias: revista de investigación y experiencias didácticas, 19(1), 151 162.

Songer N.B., Linn M.C. (1991). How do students' views of science influence knowledge integration?. Journal of research in science teaching, 28(9), 761-784.

Sutherland D., Dennick R. (2002). Exploring culture, language and the perception of the nature of science. International Journal of Science Education, 24(1), 1-25.

Tsai C.C. (2003). The interplay between philosophy of science and the practice of science education. Curriculum and Teaching, 18(1), 27-43.

Tsai C.C., Liu S.Y. (2005). Developing a multi-dimensional instrument for assessing students' epistemological views toward science. International Journal of Science Education, 27(13), 1621-1638.

Vázquez Á., Manassero M.A. (1999). Características del conocimiento científico: creencias de los estudiantes. Enseñanza de las ciencias: revista de investigación y experiencias didácticas, 17(3), 377-395.

Yin R.K. (2003). Designing case studies. En R. K. Yin (Ed.), Case study research: Design and methods (pp. 19-56). Thousand Oaks: Sage. 